\title{
Dissonância micrométrica como possibilidade analítica da música dos séculos XX e XXI
}

\author{
Leandro Gumboski \\ Universidade de São Paulo/ Instituto Federal do Paraná \\ leandro.gumboski@ifpr.edu.br
}

\begin{abstract}
Resumo: A dissonância métrica, conceito que surge no século XIX como uma ferramenta composicional, foi sistematicamente teorizada por Krebs (1999), compreendendo situações de desalinhamento métrico entre estratos interpretativos distintos. Um axioma teórico compreende que as dissonâncias métricas apresentam um estrato comum e mais rápido ou em nível menor àqueles estratos interpretativos que dissonam, chamado de estrato de pulso. Em oposição estrutural à dissonância hipermétrica, já discutida em bibliografia teórica recente, este trabalho propõe uma definição para a dissonância micrométrica. Conceituada enquanto situação em que o estrato de pulso se torna, também, um dos estratos em conflito métrico (estrato interpretativo), a dissonância micrométrica pode ser segmentada em categorias analíticas específicas. Ao teorizar três categorias possíveis, a saber, por andamento, por deslocamento e por aceleração ou retenção contínua, este trabalho ainda apresenta concisos exemplos analíticos para cada qual. Considera-se que o conceito de dissonância micrométrica é de grande relevância para se analisar o plano temporal de muitas composições dos séculos XX e XXI.
\end{abstract}

Palavras-chave: Micrométrica, Dissonância Micrométrica, Deslocamento, Andamento, Aceleração ou Retenção Contínua.

\section{Micro-Metrical dissonance as an element of music analysis of the 20th and 21 st centuries}

\begin{abstract}
Metrical dissonance is a concept that emerged in the 19th century as a compositional tool. It was systematically theorized by Krebs (1999) comprising situations of metrical non-alignment between different interpretive layers. It is a theoretical axiom that metrical dissonances present a common layer faster than those interpretive layers that dissonate, called the pulse layer. In structural opposition to hypermetrical dissonance, already discussed in recent theoretical bibliography, this paper proposes a definition for micro-metrical dissonance. Micro-metrical dissonance is conceptualized as a situation in which the pulse layer also becomes one of the layers in metrical conflict (interpretive layers). Micrometrical dissonance can be segmented into specific analytical categories, namely, speed (grouping), displacement and continuous acceleration or retention dissonance. This paper still presents concise analytical examples for each one. It is concluded that the concept of micro-metrical dissonance is of great relevance to analyze the temporal plane of many compositions of the 20 th and 21 st centuries.
\end{abstract}

Keywords: Micro-Metric, Micro-Metric Dissonance, Displacement, Speed, Continuous Acceleration or Retention.

O presente artigo desenvolve o trabalho apresentado no $\mathrm{V}$ Encontro Internacional de Teoria e Análise Musical, EITAM5 (GUMBOSKI, 2019, p. 393-406). 


\section{Dissonância métrica e micrométrica}

Das muitas especificidades teóricas concernentes à estética romântica do século XIX, a dissonância métrica figura entre aquelas de discussão ainda incipiente no meio musicológico. O conceito, que surge em meio aos escritos críticos de Hector Berlioz (1803-1869) (KREBS, 1999), para quem, ademais, tratava-se de um elemento de emprego óbvio na música de concerto de seu tempo (BERLIOZ, 2015 [1837]), foi revisitado por certo número de teóricos mais recentes.

A título de revisão, conquanto lacônica, cabe destacar Yeston (1976), que ainda se utiliza do termo "dissonância rítmica", tal qual, afinal, o compositor romântico francês. Revisando a definição de Yeston (1976), Krebs (1987) propôs inicialmente seu entendimento de dissonância métrica, o mesmo adotado no contexto deste artigo. No mesmo ano, Berry (1987), um dos primeiros a apontar para as implicações métricas das mesmas estruturas indicadas por Berlioz, Yeston e outros tantos, definiu "dissonância métrica" a partir do princípio de não congruidade vertical, um tipo de polimetria em que “a barra de compasso real é disjunta, não 'perpendicular' à 'linha' da sucessão temporal” (BERRY, 1987, p. 365).

No âmbito da dicotomia "ritmo" e "métrica", quando de estruturas dissonantes, cumpre observar ainda as reflexões de Gauldin (2004), que propõe um modelo bipartite - "dissonância rítmica" e "dissonância métrica" - cada qual com aspectos específicos. Finalmente, autor responsável pelo trabalho mais extenso já dedicado ao assunto, tornando-o a referência mais amplamente citada sobre tal, Krebs (1999) define dissonância métrica como toda estrutura que, dada sua propriedade de estratificação, apresenta algum grau de desalinhamento entre os tempos/pulsos/beats de dois ou mais diferentes estratos métricos. Partindo desta definição, o objetivo deste trabalho é apresentar algumas reflexões acerca do conceito de micrométrica e de possíveis correlações com o modelo analítico de Krebs (1999).

A teoria da dissonância métrica de Krebs (1999) está voltada, inicialmente, para a música de Schumann, estendendo-se mais naturalmente a todo o repertório romântico do século XIX, dado que o próprio conceito, ainda que em termos distintos, era de domínio de certo número de compositores deste período (BERLIOZ, 2015 [1837]; KREBS, 1999). Todavia, nota-se que, mais recentemente, a dissonância métrica tem sido revelada 
analiticamente também em repertórios dos últimos cento e vinte anos (e.g., BUTLER, 2006; PIESLAK, 2007; McCANDLLES，2010; LOVE，2013; BIAMONTE，2014; BROWN, 2014; GUMBOSKI, 2017; HANENBERG, 2018). Desta feita, ampliar o escopo de aplicação da teoria da dissonância métrica para os repertórios dos séculos $\mathrm{XX}$ e XXI implica em novas categorias inicialmente não previstas, dentre as quais está a da dissonância micrométrica.

O conceito de micrométrica não é apresentado explicitamente por Krebs, mas trazido enquanto proposta no presente trabalho, partindo-se da definição de micropulsos e de dissonâncias em nível baixo do próprio autor. Para Krebs (1999), toda dissonância métrica apresenta ao menos dois tipos de estratos de movimentação: estrato de pulso e estratos interpretativos. $\mathrm{O}$ estrato de pulso compreende o nível mais rápido e recorrente [pervasive] de movimentação métrica de um trecho musical (KREBS, 1999, p. 23), enquanto os estratos interpretativos são aqueles que exercem o estado de dissonância, i.e., aqueles estratos em conflito e desalinhamento métrico. Krebs (1999) ainda reconhece a existência de micropulsos, que englobam toda a movimentação dada por ornamentos ou pelo resultado da relação entre agrupamentos complexos e com eventos de curtas durações. Por extensão, micrométrica, portanto, compreende toda região de movimentação métrica que esteja "abaixo" - em nível menor, mais rápido - que o estrato de pulso. Por vezes, o estrato de pulso é o próprio nível de tactus (FERDAHL; JACKENDOFF, 1983), i.e., o nível de referência para ouvinte e intérprete, que tende a assumir, assim, a função de estrato primário (KREBS, 1999), o estrato percebido em primeiro plano.

Os excertos abaixo exemplificam a tipologia de níveis métricos de Krebs (1999), interpretando a rigor as definições dadas anteriormente. Na Figura 1, em "In meines Herzens Grunde”, do Oratório Paixão segundo São João, BWV 245, de J. S. Bach, não é possível inferir um estrato de micropulsos, sendo que o estrato de pulso é exercido pelo nível de semínimas, igualmente o estrato primário (tactus) deste excerto. Na Figura 2, na Sonata No. 33, Hob. XVI:20, de Haydn, o estrato de pulso assume o valor referencial de colcheia, de modo que os ornamentos presentes no trecho, bem como as passagens em fusa, compõem a movimentação em nível de micropulsos, enquanto o estrato primário tende a ser percebido pela movimentação em nível de semínimas. 
Figura 1 - Compassos iniciais do coral "In meines Herzens Grunde", do Oratório da Paixão segundo São João, BWV 245, de J. S. Bach: excerto sem movimentação em micropulsos.

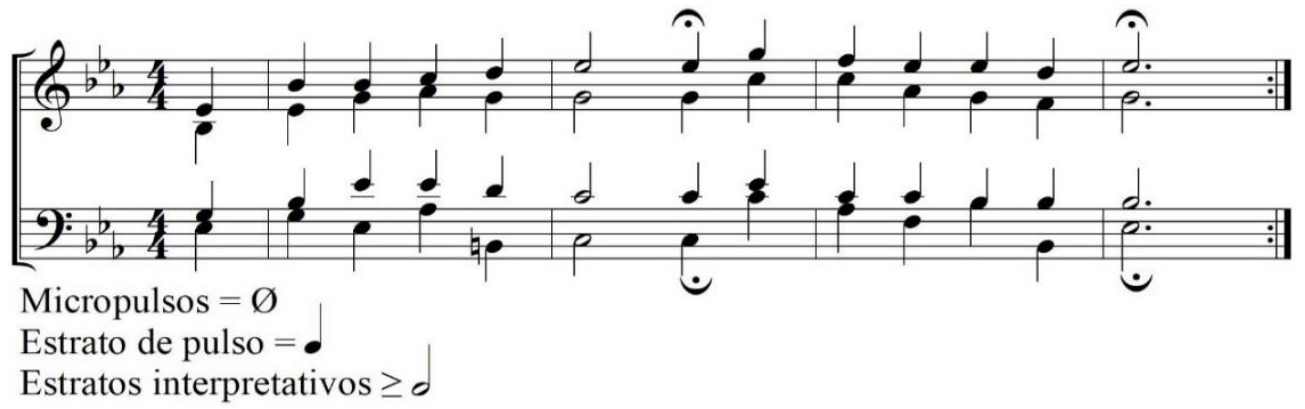

Fonte: Elaborado pelo autor.

Figura 2 - Consequente (comp. 5-8) do primeiro tema do primeiro movimento da Sonata No. 33, Hob. XVI:20, de Haydn: excerto com movimentação em micropulsos.

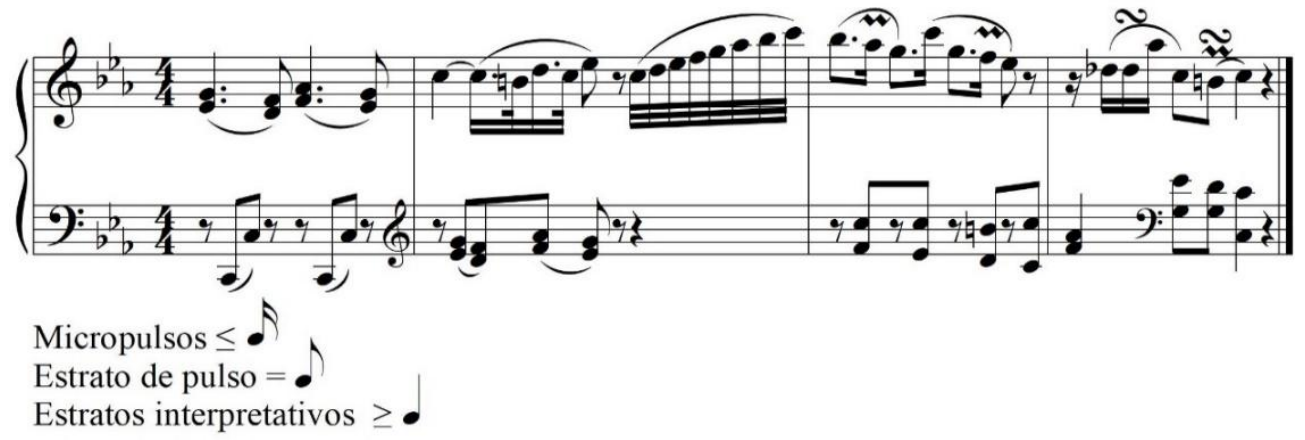

Fonte: Elaborado pelo autor.

Por fim, na Figura 3, em "Danse de la fureur, pour les sept trompettes", sexta peça de Quartuor pour la fin du temps, de Messiaen, o estrato de pulso é identificado pela movimentação em nível de colcheia, dado que, neste contexto, o estrato exercido pela figura de semicolcheia, que já opera como micropulso, não é do tipo recorrente. Neste caso o estrato primário assume uma estruturação menos isócrona.

Em tese, toda dissonância métrica ocorre pelo desalinhamento de estratos interpretativos. Os níveis em que localizamos esses estratos desalinhados é naturalmente variável. Assim, Krebs (1999) nota que dissonâncias métricas podem ocorrer em níveis alto, médio ou baixo. O princípio de dissonância em baixo nível (KREBS, 1999) inclui situações de não alinhamento entre estratos próximos do estrato de pulso. 
Figura 3 - Compassos iniciais de "Danse de la fureur, pour les sept trompetes", de Messiaen: excerto com movimentação em micropulsos.

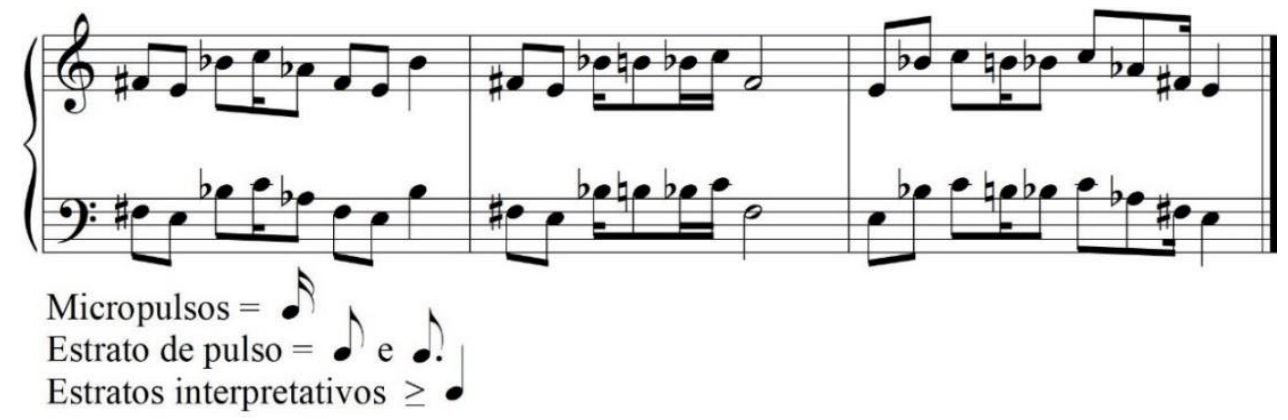

Fonte: Elaborado pelo autor.

Embora careça de mais exemplos e de um aprofundamento maior na explanação do conceito, em Santa (2019) encontramos a proposta cunhada como dissonância em nível subtactus: "aquela na qual a dissonância pode se expressar inteiramente dentro de um pulso do tactus"1 (SANTA, 2019, p. 63). Os exemplos mencionados por este autor, ao menos, correspondem também ao que se define neste artigo como dissonância micrométrica. Não obstante, devemos notar que identificar o tactus em alguns excertos musicais é um processo subjetivo e que pode variar de acordo com o ouvinte. Portanto, ambos os conceitos (dissonância em baixo nível e dissonância subtactus) aplicados ao repertório dos últimos 120 anos tornam-se menos precisos.

Fundamentando-se no conceito de micrométrica e seguindo a rigor a definição de dissonância métrica proposta por Krebs (1999), define-se, aqui, dissonância micrométrica como: qualquer estrutura que tenha algum grau de desalinhamento entre os estratos métricos, cuja relação resultante torne o próprio estrato de pulso - como estrato mais rápido e recorrente no contexto musical - um dos estratos em conflito (o estrato de pulso também se torna interpretativo). Haja vista que a micrométrica é, em certo ponto, o extremo oposto da hipermétrica, cumpre observar os apontamentos de London (2012), que identifica o limite inferior de $100 \mathrm{~ms}$ de $\mathrm{IOIs}^{2}$ na percepção humana da métrica

\footnotetext{
1 "[...] one in which the dissonance can be expressed entirely within one pulse of the tactus" (SANTA, 2019, p. 63).

2 Comumente descrito por sua acepção original em inglês, interonset interval (IOI) se refere ao intervalo de tempo entre o ataque de uma nota percebido auditivamente e o início da próxima. London (2012) explana de modo consistente sobre os limites da percepção humana descritos em termos de IOIs: no caso da métrica musical, esses limites estão, conforme o autor, entre, aproximadamente, $100 \mathrm{~ms}$ e $5.000 \mathrm{~ms}$ de IOIs.
} 
musical. Nota-se, doravante, que trechos musicais em dissonância micrométrica tendem a soar, em certa medida, como amétricos, uma vez que a relação entre os estratos desalinhados se torna extremamente complexa e de difícil percepção auditiva. Assim como no caso das dissonâncias hipermétricas, para as quais cabem críticas sob a perspectiva de teorias da cognição musical como um constructo não mais percebido auditivamente como métrico (LONDON, 2012), as dissonâncias micrométricas também aparentam se esvaziar de sua qualidade básica primária - serem percebidas como estruturas métricas. No entanto, reitero os apontamentos de Brower (1993) quanto às distintas estratégias de escuta (captura [entrainment] e contagem [counting]): a estratégia de captura, como ferramenta da percepção auditiva, em sua versão essencialmente qualitativa, é utilizada na escuta de dissonâncias micrométricas, enquanto o aspecto quantitativo, exibido nos exemplos analíticos, é alcançado por outros meios (em geral, a própria partitura da peça).

Constata-se que o conceito de dissonância micrométrica proposto aqui não se configura apenas como um termo diferente para identificar o mesmo fenômeno já previsto por Krebs (1999 - dissonância métrica em baixo nível), mas sugerimos um novo nível de análise, ou seja, do mais baixo para o mais alto: micrométrico, (métrico) de baixo nível, médio e alto nível ou hipermétrico. Neste contexto, entende-se a dissonância de baixo nível como sendo caracterizada pelo não alinhamento entre os estratos imediatamente acima do estrato de pulso. Uma dissonância em nível médio seria aquela em que o estrato primário não é o estrato imediatamente acima do estrato de pulso, ainda que seja um estrato em conflito.

A observação destas distinções em exemplos analíticos é importante neste momento. Os primeiros compassos de "Die Rose, Die Lilie, die Taube", terceira peça de Dichterliebe, Op. 48, de Robert Schumann, são analisados por Krebs (1999, p. 161) como dissonância de baixo nível, o que está de acordo com o que propomos como dissonância métrica. A Figura 4 apresenta os compassos iniciais deste lied, demonstrando que o estrato de pulso em nível de semicolcheia é um estrato alinhado com os dois estratos imediatamente superiores, ambos em nível de colcheia, desalinhados entre si. 
Figura 4 - Compassos iniciais de "Die Rose, Die Lilie, die Taube", terceiro Lied de Dichterliebe, Op. 48, de R. Schumann: excerto com dissonância métrica por deslocamento em baixo nível.

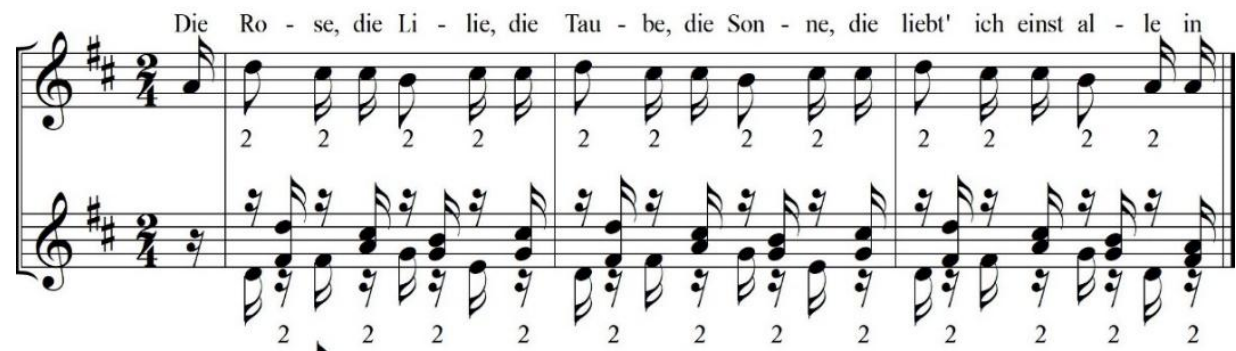

Estrato de pulso $=$

Estratos interpretativos $\geq$

Fonte: Elaborado pelo autor.

Porém, se tomarmos "Eusebius", quinta peça do Carnaval, Op. 9, como exemplo também citado por Krebs (1999, p. 53) como dissonância de baixo nível -, veremos que essa passagem é especificamente diferente das outras, pois é uma dissonância micrométrica. A Figura 5 demonstra que o estrato métrico mais rápido, que exerceria a função de estrato de pulso, em nível de septina de colcheia, está em conflito com outro estrato superior, mais lento, em nível de semínimas, o que caracteriza, fundamentalmente, uma dissonância micrométrica.

Figura 5 - Compassos iniciais de "Eusebius", do Carnaval, Op. 9, de R. Schumann: excerto em dissonância micrométrica.

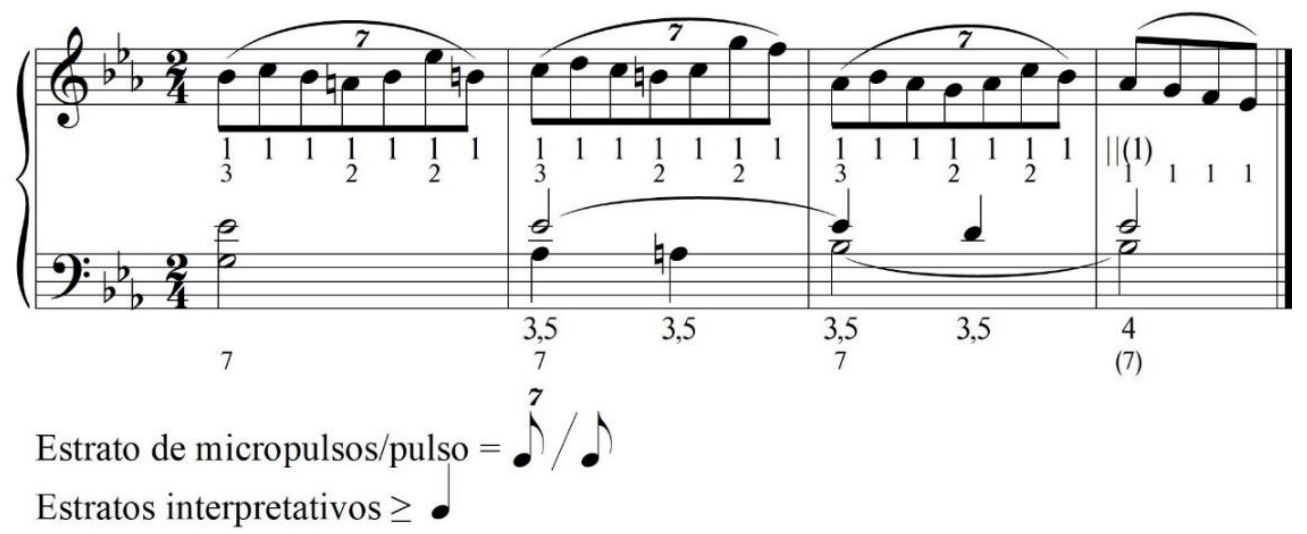

Fonte: Elaborado pelo autor. 
Uma das consequências imediatas do conceito de dissonância micrométrica é um detalhamento ainda maior da taxonomia de dissonâncias. Nas seções seguintes, detalharemos nossa proposta para as especificidades das relações por andamento $\left(\mathrm{D}_{m}: \mathrm{A}\right)$ e por deslocamento $\left(\mathrm{D}_{m}: \mathrm{D}\right)$, em situações micrometricamente dissonantes. Há, ainda, uma terceira categoria cujas implicações são necessariamente micrométricas: por aceleração ou retenção contínua $\left(\mathrm{D}_{m}: \mathrm{ARC}\right)$.

\section{Dissonância micrométrica por andamento}

Destaca-se, inicialmente, a possibilidade definida aqui como dissonância micrométrica por andamento. Admitida enquanto possibilidade por extensão ao conceito de dissonância por agrupamento (grouping dissonance) de Krebs (1999), trata-se uma dissonância ocasionada por estratos representados por números não múltiplos. Todavia, $\mathrm{D}_{m}$ :A implica em uma dissolução do estrato de pulso, transferindo-lhe a função de estrato interpretativo, como na Figura 6. Visualmente, observa-se que, mantendo-se os princípios do modelo de notação analítica de Krebs (1999) - em que o IOI do estrato mais baixo (estrato de pulso, em dissonâncias métricas) assume o valor da unidade de referência (1) para os estratos interpretativos,$- \mathrm{D}_{m}$ :A implica em agrupamentos com cardinalidades expressas por números não inteiros (as diferenças de andamentos que não são múltiplos entre os estratos desalinhados são observáveis em relação ao nível métrico mais rápido também).

Por praticidade, o estrato que assume o valor 1 como unidade de referência pode, convencionalmente, ser sempre o estrato mais rápido, independente do contexto e da seção que se analisa - este é o caso do exemplo hipotético da Figura 6. Todavia, há maior funcionalidade analítica se se atenta para o contexto do trecho musical em pauta, mormente pelo princípio de métrica como processo (HASTY, 2020), de modo que o conjunto de informações musicais completas no passado ajudam a determinar a análise que se faz do trecho presente - este é o exemplo dado pela Figura 7, demonstrando um hipotético processo métrico de uma estrutura metricamente dissonante para outra micrometricamente dissonante (na realidade, as mesmas indicadas como exemplo da Figura 6). 
Figura 6 - Acima: dissonância métrica por andamento; abaixo: dissonância micrométrica por andamento.
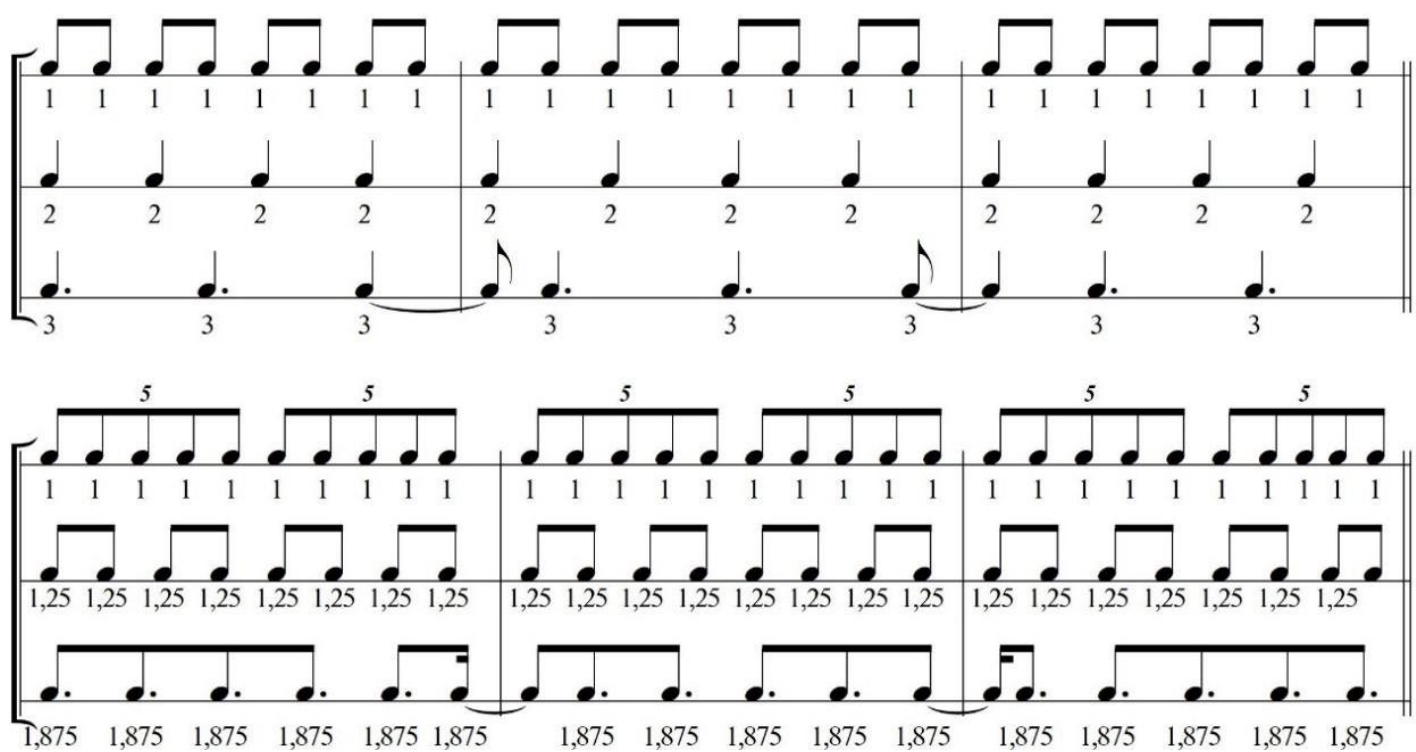

Fonte: Elaborado pelo autor.

Figura 7 - Processo métrico de $\mathrm{D}: \mathrm{A} \rightarrow \mathrm{D}_{m} \mathrm{~A}$ : figura de colcheia se mantém como referência de unidade 1.

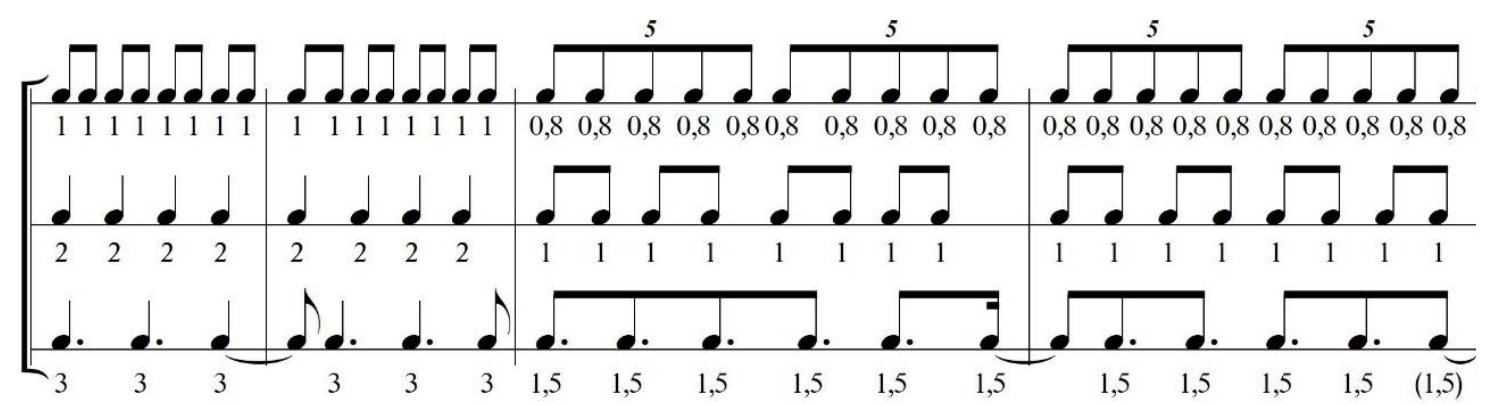

Fonte: Elaborado pelo autor.

A título de exemplo, mencionamos curtos excertos de 3 peças: "Pórtico do Crepúsculo", primeira peça de Cartas Celestes de Almeida Prado; "To Begin" de Living Room Music de John Cage; e Lamentos do Morro de Garoto, no arranjo de Lucas Telles para o álbum de Túlio Araújo. Os três apresentam notação tradicional utilizando do recurso de quiálteras, que facilita a exemplificação e a identificação da relação micrometricamente dissonante. 
As primeiras seções do movimento "Pórtico do Crepúsculo", da obra Cartas Celestes (1974) de Almeida Prado, apresentam uma série de "quadros cronométricos" com fragmentos melódicos que devem se repetir precisamente pelo tempo indicado na partitura. A sobreposição entre as linhas da mão direita e esquerda ao piano gera um desalinhamento - dissonância - a partir de agrupamentos de quiálteras de cinco fusas (mão direita) e agrupamentos de quatro fusas (mão esquerda) (Figura 8). O resultado é deveras textural, mas trata-se de uma dissonância micrométrica por andamento $\left(\mathrm{D}_{m} \mathrm{~A}\right)$. Outrossim, cabe notar que a composição já se inicia em estado de dissonância micrométrica; é um caso em que não há como inferir, com facilidade por uma escuta "pura", o estrato de pulso.

Figura $8-\mathrm{D}_{m} \mathrm{~A}$ no início de "Pórtico do Crepúsculo" de Almeida Prado.

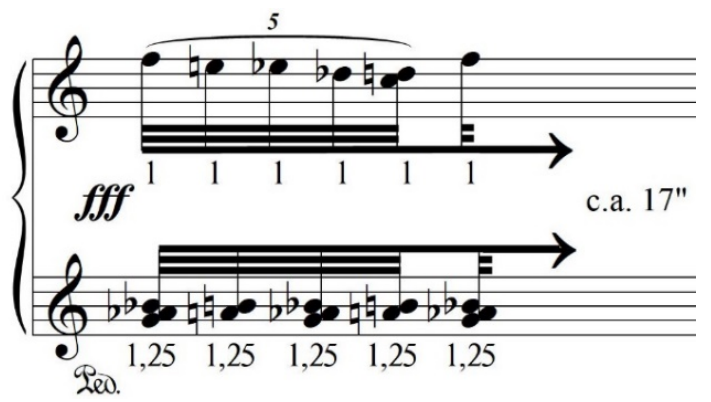

Fonte: Elaborado pelo autor.

“To Begin” é o primeiro movimento da composição Living Room Music (1940) de John Cage. A obra, na íntegra, é repleta de situações similares à ilustrada na Figura 9. Aqui temos a exemplificação de um processo métrico que pode ser notado como $\mathrm{D}: \mathrm{A} \rightarrow$ $\mathrm{D}_{m} \mathrm{~A}$ - leia-se, dissonância métrica por andamento torna-se dissonância micrométrica por andamento. $\mathrm{O}$ excerto demonstrado na Figura 9 corresponde aos compassos 13 a 18 de "To Begin", de modo que o compasso 14 introduz uma situação micrometricamente dissonante no trecho em questão. 
Figura 9-D:A $\rightarrow \mathrm{D}_{m} \mathrm{~A}$, c. 13-18 de "To Begin" de Living Room Music, de John Cage.

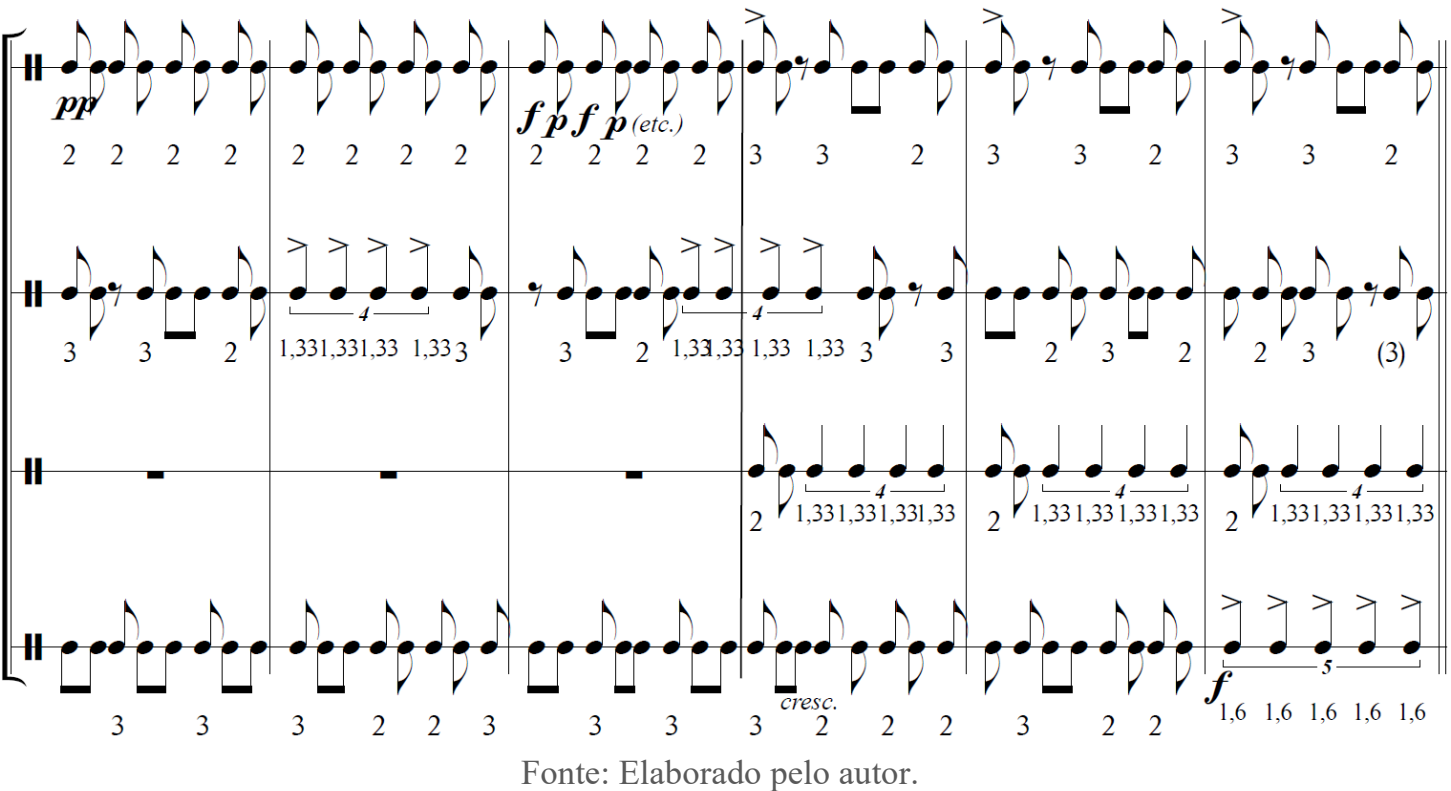

Por fim, Lamentos do Morro, choro composto por Garoto, recebeu um arranjo recente do violonista Lucas Telles, registrado sonograficamente no álbum Monduland (2017) de Túlio Araújo. A peça, integralmente desenvolvida com um dueto entre violão e pandeiro, inicia com um processo de consonância métrica para dissonância micrométrica por andamento, cuja notação simbólica poderia ser: $\mathrm{C} \rightarrow \mathrm{D}_{m} \mathrm{~A}$. Na Figura 10 é possível notar este processo métrico que ocorre no terceiro compasso.

Figura $10-\mathrm{C} \rightarrow \mathrm{D}_{m} \mathrm{~A}$ no início de Lamentos do Morro, de Garoto, com arranjo de Lucas Telles.

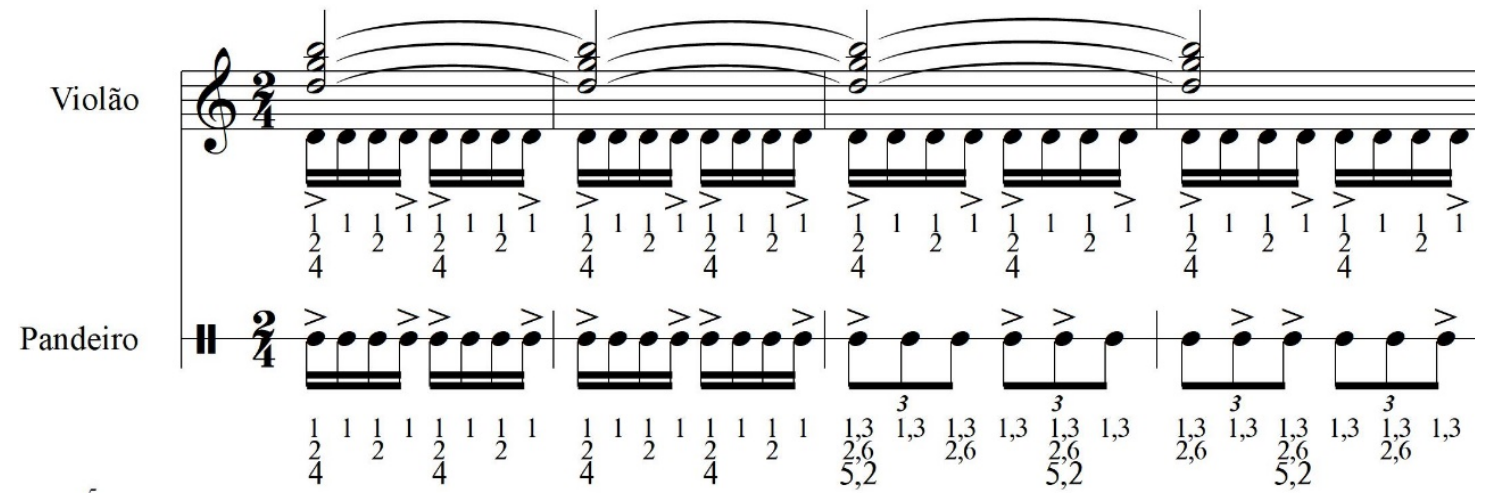

Fonte: Elaboração do autor a partir de partitura cedida por Lucas Telles. 


\section{Dissonância micrométrica por deslocamento}

A dissonância micrométrica por deslocamento $\left(\mathrm{D}_{m} \mathrm{D}\right)$ é admitida enquanto possibilidade por extensão da dissonância métrica por deslocamento (displacement dissonance) (KREBS, 1999), identificada em situação de não alinhamento entre estratos que operam no mesmo nível métrico e possuem a mesma organização interna, mas estão deslocados temporalmente entre si. $\mathrm{D}_{m} \mathrm{D}$, por sua vez, implica em deslocamento em relação ao próprio estrato de pulso, como na Figura 11, possibilitando inclusive a sensação de dois ou mais estratos de pulso desalinhados e concomitantes, ou, pelo contrário, a falta de identificação de um estrato de pulso, em virtude da relação complexa e ambígua entre eles. Assim como no caso de $\mathrm{D}_{m} \mathrm{~A}$, em que as análises revelam relações decimais entre os estratos métricos, uma $\mathrm{D}_{m} \mathrm{D}$ também revelada pelo deslocamento em valor menor do que o da própria unidade 1 do estrato de pulso. Na Figura 11, por exemplo, os deslocamentos dos estratos são em valores de 0,5 e 0,75 .

Figura 11 - Acima: dissonância métrica por deslocamento; abaixo: dissonância micrométrica por
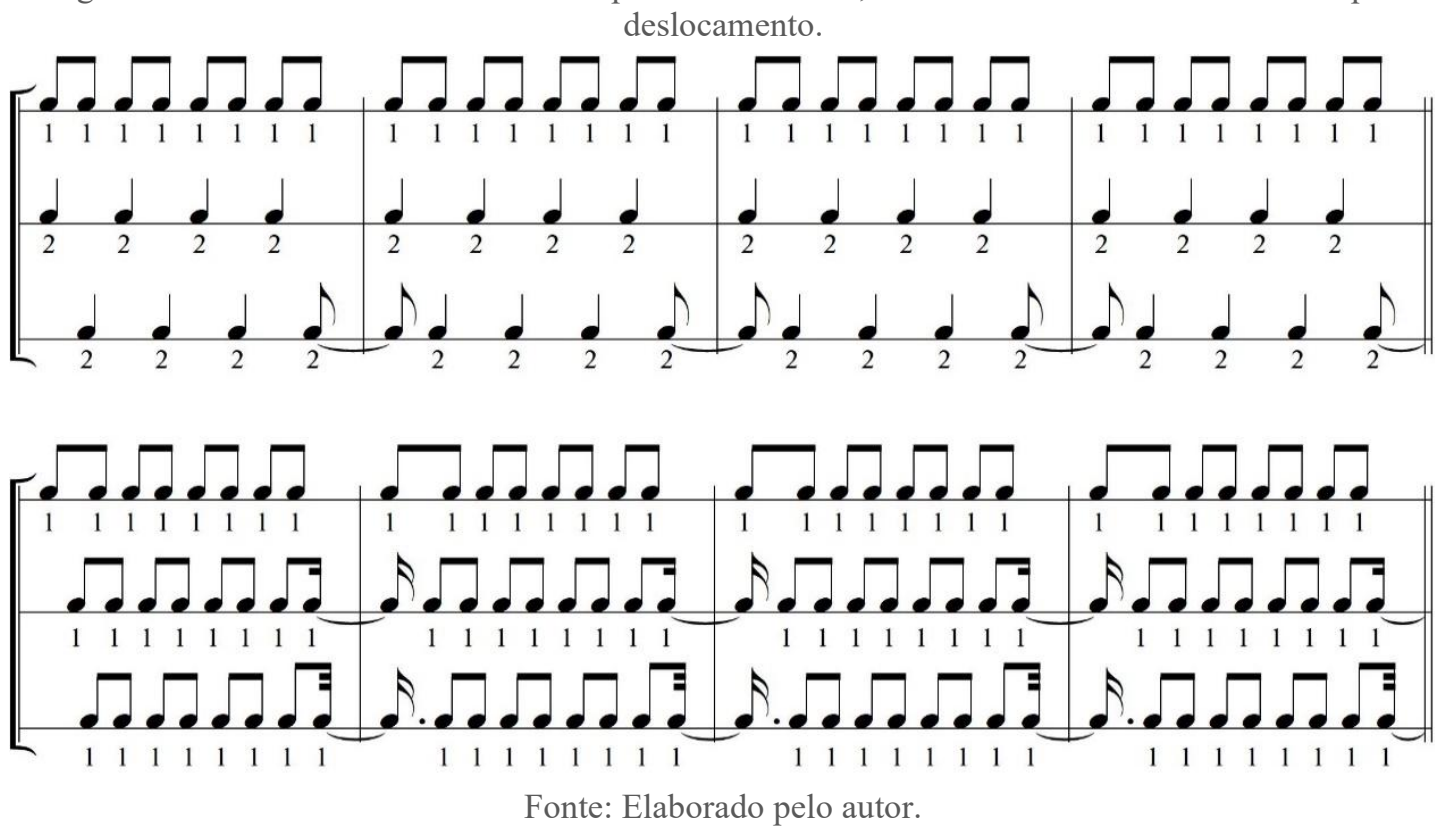

A título de exemplo, no Prelúdio No. 3 (1929) de Messiaen - "Le nombe léger" -, temos uma situação de dissonância micrométrica por deslocamento $\left(\mathrm{D}_{m} \mathrm{D}\right)$. Na Figura 12 
é possível notar que o estrato de pulso dos primeiros compassos da peça é exercido a nível de colcheia, de modo que o deslocamento em valor de semicolcheia entre os estratos da mão direita e mão esquerda ao piano já opera a nível micrométrico. A notação rítmica utilizada na Fig. 12 não é a original - ela foi adaptada de modo a facilitar a visualização da dissonância micrométrica indicada.

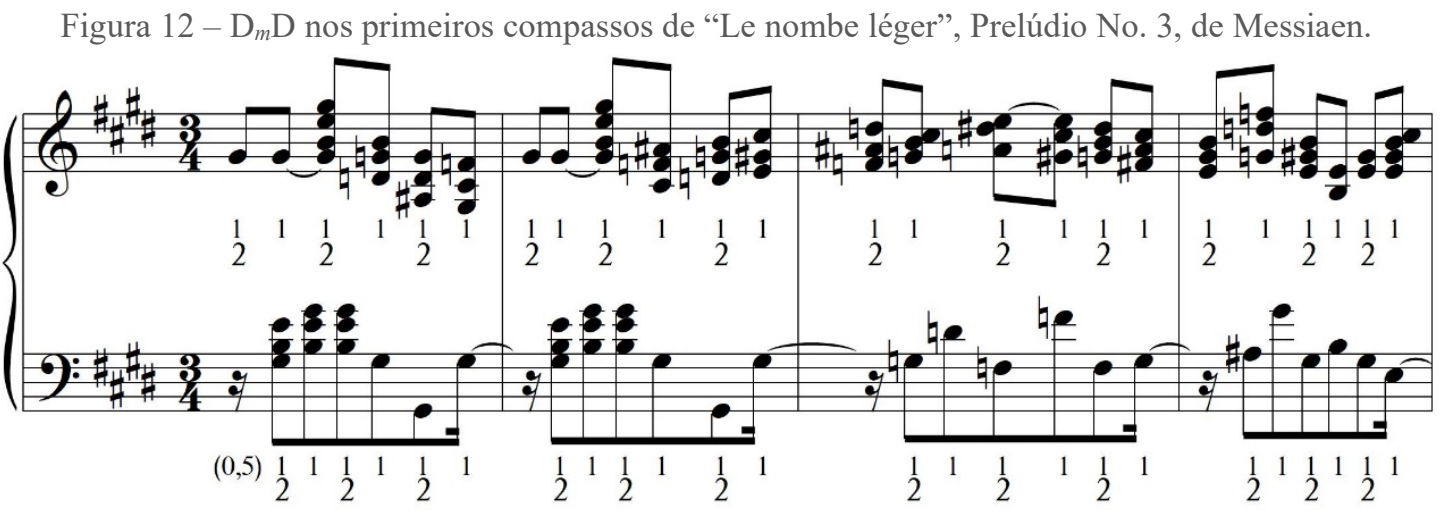

Fonte: Elaborado pelo autor.

\section{Dissonância micrométrica por aceleração ou retenção contínua}

Há uma terceira categoria a ser observada, cujo conceito apresenta certo ineditismo teórico: dissonância micrométrica por aceleração ou retenção contínua ( $\left.\mathrm{D}_{m}: \mathrm{ARC}\right)$. Observe-se que as dissonâncias por andamento (métricas ou micrométricas), apresentam como característica comum o constante realinhamento dos estratos em desalinhamento (o início dos estratos é conjunto e a resolução da dissonância é previsível por um processo de minimização). Já as dissonâncias por deslocamento (métricas ou micrométricas) têm como característica o início já deslocado de um estrato em relação a outro(s), de modo que, sem alterações na estrutura de algum dos estratos, o realinhamento jamais acontece. Uma $\mathrm{D}_{m}: \mathrm{ARC}$, portanto, se caracteriza pela interação entre estratos distintos com estruturação interna que pode ser similar, mas, contrariamente à $\mathrm{D}_{m}: \mathrm{D}$, o que gera $o$ desalinhamento em nível de micrométrica são as diferentes acelerações ou retenções contínuas na execução de cada estrato. 
Utilizando uma metáfora com a física mecânica clássica, dissonâncias por andamento (D:A ou $\left.\mathrm{D}_{m}: \mathrm{A}\right)$ são casos em que dois ou mais objetos apresentam acelerações constantes, mas velocidades distintas, e essas velocidades são representadas por números não múltiplos. Identificamos que a situação é micrométrica quando a proporção entre as velocidades desses objetos é representada por números decimais, incluindo o objeto que se move na velocidade mais rápida. Porém, é musicalmente possível, analogamente, imaginarmos dois objetos com acelerações variáveis, sendo que as velocidades resultantes são também distintas. Para esses casos, propomos o conceito de $\mathrm{D}_{m}$ :ARC. Considerando a velocidade variável de ao menos um dos estratos, não há como notar com precisão a relação matemática entre eles pelo sistema tradicional da teoria da dissonância métrica, mas seria necessário um gráfico que demonstrasse a variação de velocidade, como no exemplo hipotético da Figura 13: uma dissonância micrométrica composta - 3 estratos desalinhados (KREBS, 1999) - por aceleração ou retenção contínua.

Figura 13 - Gráfico de uma $\mathrm{D}_{m} \mathrm{ARC}$ hipotética: estrato 1 com aceleração constante; estrato 2 com aceleração contínua; estrato 3 com retenção contínua - consonância métrica aos 30s.

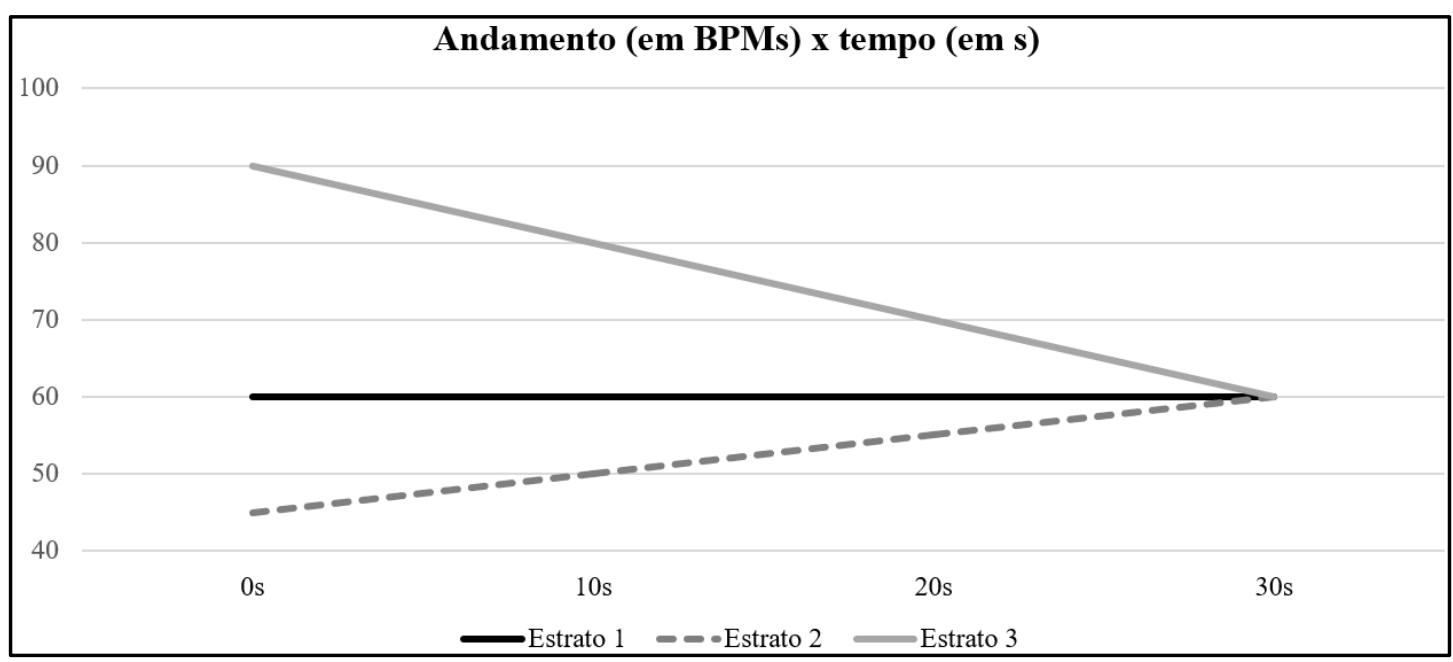

Fonte: Elaborado pelo autor.

Lembremos das tantas composições que se utilizam dos processos de phase shifting descritos por Steve Reich (2004). Os trechos de transição entre cada seção em Piano Phase (1967), por exemplo, caracterizam dissonâncias micrométricas por aceleração ou 
retenção contínua $\left(\mathrm{D}_{m} \mathrm{ARC}\right)$. Aqui, o estrato de pulso é estabelecido logo no início da peça e, junto com ele, um estado de consonância primária (KREBS, 1999); a dissonância micrométrica, portanto, é introduzida gradualmente, sem que se desfaça o estrato de pulso original - esta é uma situação em que o estrato de pulso, inicialmente em contexto de consonância, torna-se, também, um dos estratos em conflito (interpretativo). A Figura 14 apresenta um excerto analítico do início da peça, progredindo de um estado de consonância primária, para uma dissonância micrométrica por aceleração contínua, até alcançar uma dissonância métrica por deslocamento. Note-se, assim, que peças como Clapping Music (1972), que também exploram o processo de phase shifting, operam apenas por dissonâncias métricas, e não micrométricas.

Figura 14 - Processos métricos no início de Piano Phase de Steve Reich: $\mathrm{C} \rightarrow \mathrm{D}_{m}: \mathrm{ARC} \rightarrow \mathrm{D}_{m} \mathrm{D}$.

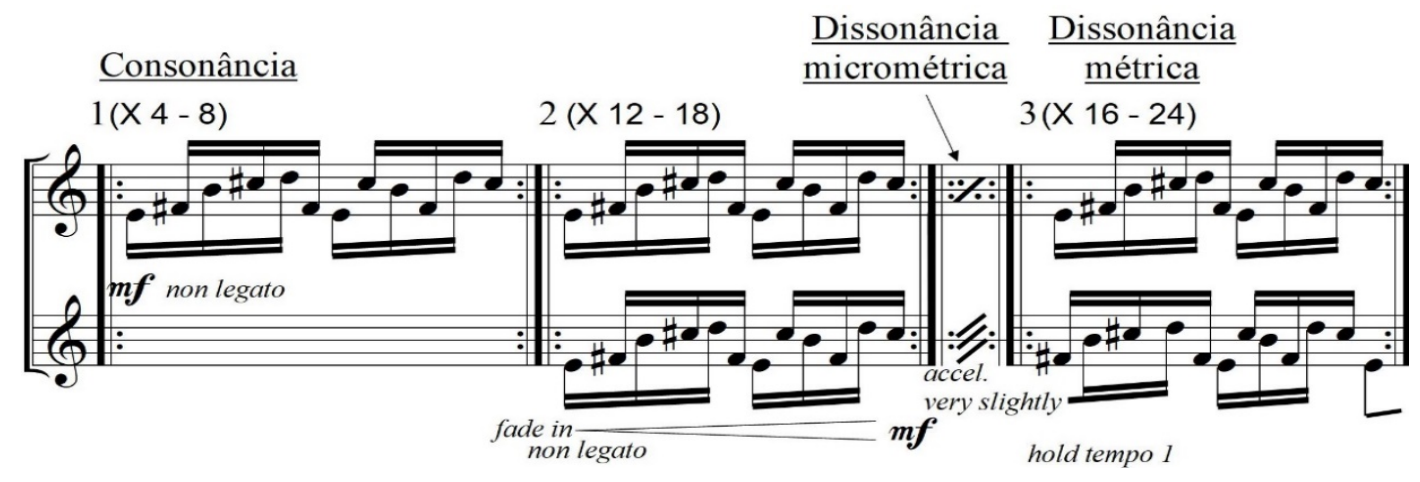

Fonte: Elaborado pelo autor.

Curiosamente, há certo número de casos similares - micrométrica por aceleração ou retenção contínua - na música do século XX. Um segundo exemplo a ser citado encontra-se na extensa lista de estudos para pianola compostos por Nancarrow. O Study No. 37 (1965-69) é composto por uma série de doze cânones, cada qual integrado por doze partes em andamentos distintos resultantes de acelerações e retenções de tempo. $\mathrm{O}$ cânone n. 7, por exemplo, apresenta a exposição de todos os doze estratos conjuntamente, mas, como as oscilações de andamentos são diferentes, os estratos compõem uma estrutura cada vez mais desalinhada micrometricamente em seu próprio devir. Cumpre observar que Nancarrow propôs, em meio às suas práticas composicionais, o conceito de 
“dissonância temporal” para definir o tipo de estruturação métrica que ele próprio utilizou (THOMAS, 2000), mas pelas definições que propomos neste trabalho, o conceito de Nancarrow se divide em duas situações conceituais: dissonância micrométrica por andamento (em estruturas similares à do Poème Symphonique de Ligeti, 1962) e dissonância micrométrica por aceleração e retenção contínua.

\section{Considerações finais}

A partir dos exemplos analíticos apresentados ao longo deste artigo, é possível observar que o conceito de dissonância micrométrica pode ser utilizado para se analisar certo número de composições dos séculos XX e XXI, ajudando na compreensão de alguns processos temporais. A partir de um número maior de análises mais aprofundadas, para muito além dos exemplos lacônicos apresentados anteriormente, é possível mapear proximidades estilísticas ou salientar aquelas já feitas por determinados aspectos entre compositores e/ou escolas distintas.

No entanto, é preciso ressaltar que a dissonância micrométrica não é absolutamente exclusiva dos repertórios do século XX. A condição básica posta no conceito apresentado ao início deste artigo - o estrato de pulso ser, também, um dos estratos em conflito - é encontrada em certo número de exemplos da música de concerto do século XIX. Casos similares ao de "Eusebius", de Schumann, citado no início deste artigo, que já demonstram a existência de dissonâncias micrométricas, ainda que em relações mais simples entre os estratos, são encontrados também em composições de Brahms. Todavia, é em certas correntes estéticas dos séculos XX e XXI que percebemos uma recorrência muito maior de estruturas similares, empregadas também de modo mais intenso e complexo. A partir do desenvolvimento de novos trabalhos analíticos será possível compreender a dimensão da dissonância micrométrica como elemento estrutural em repertórios dos séculos XX e XXI. 


\section{Referências}

BERLIOZ, Hector. Strauss: Son Orchestre, Ses Valses - De L'Avenir Du Rhythme. Site Hector Berlioz, 2015 [Journal de Débats, p. 1, 10 nov. 1837]. Disponível em: http://www.hberlioz.com/feuilletons/debats371110.htm. Acesso em: 8 jun. 2021.

BERRY, Wallace. Structural Functions in Music. Mineola: Dover Publications, 1987.

BIAMONTE, Nicole. Formal Functions of Metric Dissonance in Rock Music. Journal of Society for Music Theory, v. 20, n. 2, [s.n.], 2014.

BROWN, Ryan. From EDM to Math Rock: Metrical Dissonance in the Music of Battles. Tese (Doutorado) - University of Princeton, Princeton, NJ, 2014.

BROWER, Candace. Memory and Perception of Rhythm. Music Theory Spectrum, v. 15, n. 1, p. 19-35, 1993.

BUTLER, Mark J. Unlocking the Groove: Rhythm, Meter, and Musical Design in Electronic Dance Music. Bloomington and Indianapolis: Indiana University Press, 2006.

GAULDIN, Robert. Harmonic Practice in Tonal Music. New York: W. W. Norton, 2004.

Gumboski, Leandro. Da dissonância micrométrica: possibilidades teórico-analíticas. In: ENCONTRO INTERNACIONAL DE TEORIA E ANÁLISE MUSICAL, 5., 2019, Campinas. Anais... Campinas, Universidade Estadual de Campinas, 2019. p. 393-406. Disponível em: https://eitam5.nics.unicamp.br/wp-content/uploads/2021/01/EITAM5-paper_28_GumboskiLpp 393-406.pdf. Acesso em: 18 maio 2020.

HANENBERG, Scott James. Unpopular Meters: Irregular Grooves and Drumbeats in the Songs of Tori Amos, Radiohead, and Tool. Tese (Doutorado) - Faculty of Music, University of Toronto, 2018.

HASTY, Christopher F. Meter as rhythm. 20th anniversary edition. New York: Oxford University Press, 2020.

KREBS, Harald. Fantasy Pieces: metrical dissonance in the music of Robert Schumann. New York: Oxford University Press, 1999.

LONDON, Justin. Hearing in Time: Psychological Aspects of Musical Meter. 2. ed. New York: Oxford University Press, 2012.

LOVE, Stefan Caris. Subliminal Dissonance or "Consonance"? Two Views of Jazz Meter. Music Theory Spectrum, v. 35, n. 1, p. 48-61, 2013.

McCANDLLES, Gregory Richard. Rhythm and Meter in the Music of Dream Theater. Tese (Doutorado) - College of Music, The Florida State University, Tallahassee, 2010.

PIESLAK, Jonathan. Re-casting Metal: Rhythm and Meter in the Music of Meshuggah. Music Theory Spectrum, v. 29, n. 2, p. 219-245, 2007.

REICH, Steve. Music as a Gradual Process. In: COX, Christoph; WARNER, Daniel (Org.). Audio Culture: Readings in Modern Music. London: Bloomsburry, 2004 
SANTA, Matthew. Hearing Rhythm and Meter: Analyzing Metrical Consonance and Dissonance in Common-Practice Period Music. London and New York: Routledge, 2019.

YESTON, Maury. The Stratification of Musical Rhythm. New Haven and London: Yale University Press, 1976. 